\title{
Magnetic forces between arrays of cylindrical permanent magnets
}

\author{
D. Vokoun ${ }^{\mathrm{a}, *}$, G. Tomassetti ${ }^{\mathrm{b}}$, M. Beleggia ${ }^{\mathrm{c}}$, I. Stachiv $^{\mathrm{a}}$ \\ a Institute of Physics, Academy of Science of the Czech Republic, Prague, Czech Republic \\ b Dipartimento di Ingegneria Civile, Università degli Studi di Roma “Tor Vergata”, Rome, Italy \\ c Center for Electron Nanoscopy, Technical University of Denmark, DK-2800 Kgs. Lyngby, Denmark
}

\section{A R T I C L E I N F O}

\section{Article history:}

Received 12 June 2010

Received in revised form

10 August 2010

Available online 20 August 2010

Keywords:

Permanent magnet

Cylindrical magnet

Attraction force

Magnetostatic interaction

\begin{abstract}
A B S T R A C T
Permanent magnet arrays are often employed in a broad range of applications: actuators, sensors, drug targeting and delivery systems, fabrication of self-assembled particles, just to name a few. An estimate of the magnetic forces in play between arrays is required to control devices and fabrication procedures. Here, we introduce analytical expressions for calculating the attraction force between two arrays of cylindrical permanent magnets and compare the predictions with experimental data obtained from force measurements with $\mathrm{NdFeB}$ magnets. We show that the difference between predicted and measured force values is less than $10 \%$.
\end{abstract}

(c) 2010 Elsevier B.V. All rights reserved.

\section{Introduction}

Permanent magnets may be grouped into arrays to adjust their mutual magnetostatic interaction and consequently, the force acting upon them. Recently, permanent magnet arrays have been utilized in many applications; among others: eddy current dampers [1,2], magnetic refrigerators [3,4], micropumps [5]. Furthermore, the repulsive force between permanent magnets and superconducting samples is utilized for levitation [6]. In the literature related to permanent magnets, calculations of forces between magnets of various shapes and geometries have their relevance in the context of several applications. For example, in Ref. [7], the authors model magnetic bandages for drug targeting. In Ref. [8], the authors take advantage of inter-particle magnetostatic interactions for selfassembly of floating magnetic particles, with the perspective of employing them in photonic band gap materials fabrication. Magnetic forces in self-assembled arrays are studied in Ref. [9]. Magnetostatic interactions and forces between two magnetic nanotubes are examined in Ref. [10].

In our previous study [11] we calculated the attraction force between two groups of cylindrical permanent magnets for the purpose of designing magnetic fasteners. However, in [11] the number of acting permanent magnets in either set was limited to four. In the present study we extend the formalism to cope with much larger arrays, and discuss their mean features in terms of arising forces and optimization of geometrical parameters (mainly: cylinders' aspect ratios and distances involved). In our model, we assume that all the cylindrical magnets are axially magnetized and

\footnotetext{
* Corresponding author.

E-mail address: vokoun@fzu.cz (D. Vokoun).
}

all the cylinder axes are parallel, while the cylinders may be laterally and vertically displaced. In Ref. [12] the author derived the expression for the interaction between two magnetic bodies consisting of identical cylindrical magnets arranged in a regular square grid. We adopt this methodology to predict the forces in play between two arrays of magnetized cylinders.

In this manuscript we review the formula for the attraction force between two sets of cylindrical magnets described in Ref. [11]. Then we calculate the force between two magnetic bodies consisting of identical cylindrical magnets arranged in regular grids. The magnet centers of either set are coplanar while the number of magnets of one of the set is so large to be considered infinite. Finally, we show the measured dependence of force versus distance between two sets containing $2 \times 2$ and $6 \times 6$ magnets, respectively, and discuss the results. If we exclude the data point when magnets are in contact, the acquisition of which is problematic (see discussion below), the measurements and calculations are in excellent agreement in terms of force decay with distance (reflecting the quadrupole-quadrupole type of interaction present in our geometry), while the discrepancy between calculated and measured force values does not exceed $10 \%$. We observe a systematic discrepancy, as predictions always overestimate measurements. The possible reasons for such systematic trend are discussed in the following.

\section{Theoretical framework}

2.1. Force between two axially magnetized cylindrical magnets with their axes parallel

The attraction force between two axially magnetized cylindrical magnets introduced in [11] is based on an assumption of uniform 
magnetization of the magnets. The assumption may be fulfilled in permanent magnets with high magnetic anisotropy, or in presence of strong applied fields. In Ref. [11] we showed that, in spite of the seemingly unrealistic assumption of uniform magnetization, the measured force between two NdFeB cylindrical magnets was in excellent agreement (below 5\%) with that calculated using our expressions.

The attraction force between two cylinders in the axial direction is obtained as derivative of the total magnetostatic interaction energy $E$ with respect to the axial coordinate, that we choose to name $z$. In [11], we derived a formula for the attraction force between two identical, axially magnetized cylinders, with their axes parallel but laterally displaced

$F_{z}^{1 / 1}(\rho, \zeta, \tau)=-F_{0} \int_{0}^{+\infty} J_{0}(\rho q) \frac{J_{1}^{2}(q)}{q} \sinh ^{2}(q \tau) e^{-q \zeta} d q$

where $F_{0}=4 \pi \mu_{0} M^{2} R^{2}$ reflects the order of magnitude of the forces involved (for the magnets used in this study, $F_{0}=42.6 \mathrm{~N}$ ), $M$ is the saturation magnetization of the material, $R$ and $t$ are the cylinder radius and height, respectively, $\tau=t /(2 R)$ is the cylinder aspect ratio, $\rho=r / R$ and $\zeta=z / R$ are the reduced lateral and axial distances between the centers of the two cylinders, respectively, and $J_{0}(q)$, $J_{1}(q)$ are Bessel functions of first kind [13]. The negative sign in Eq. (1) indicates an attractive force. If the cylinders' magnetizations are anti-parallel, the sign of Eq. (1) is reversed, as force is repulsive. For long cylinders $(\tau \gg 1)$, the contact $(\rho=0, \zeta=2 \tau)$ force is $F_{0} / 8$.

\subsection{Force between two finite arrays of cylindrical magnets}

We express the attraction force between two sets of magnets, each set consisting of identical cylindrical magnets of radius $R$ and height $t$. In each set the magnets are firmly connected together forming a magnetic body. The magnets' centers in either set are located over a square lattice, with lattice parameter $2 R$ (each magnet touches at least two other magnets in lateral directions). The two arrays are vertically displaced by an amount $z \geq t$, where $z$ is the distance between the two planes containing the magnets' centers. The two grids are aligned and centered: no lateral displacement is in effect, and cylinders in either set are coaxial. See Fig. 1.

We first assume that one set comprises four magnets whereas the other set consists of $2 N \times 2 N$ magnets $(N>0)$. In either set, firstneighbor magnets have their magnetization anti-parallel (Fig. 1 ). The advantage of the anti-parallel magnetization arrangement is its stability - the magnets do not repel each other. Furthermore, such arrangement features an interesting property: the attraction force between the two sets can be larger than four times the attraction force between just two of the magnets in the set. As a complement to the anti-parallel arrangement we also intend to mention the case of parallel magnetization arrangement.

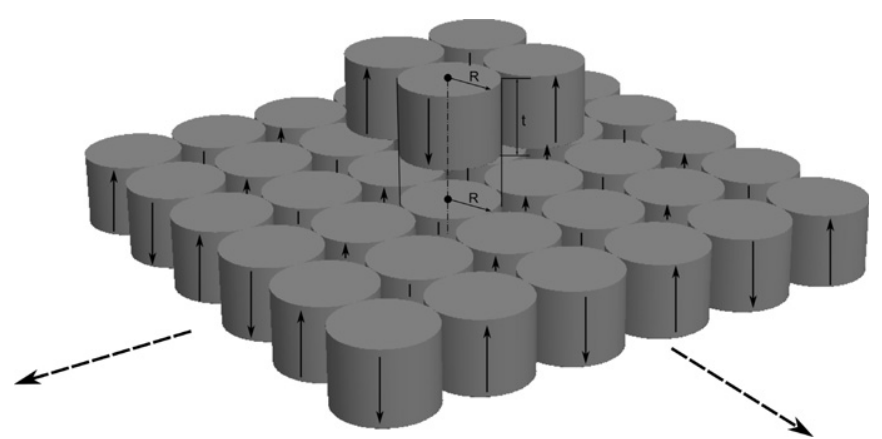

Fig. 1. A scheme of two sets of cylindrical magnets. One set is formed by the four upper magnets whereas the other set is formed by the bottom magnets and may tend to be infinite (see the dashed arrows).
The calculation of the attraction force requires knowledge of the total magnetostatic interaction energy of the system. We have to examine the interaction energy of each possible couple of magnets, one in each set. The interaction energy within each set is not relevant, as it does not depend on $z$. Since the magnets are located over lattice points, the lateral position of each magnet can be specified by two indexes $(i, j)$, meaning that the center of the magnet in question lies over the $i$-th row and $j$-th column of the larger grid. Because of symmetry, in the set of four magnets we consider only that with coordinates $(N, N)$, and then multiply the energy by four. The total energy is then

$E=4 \sum_{i=1}^{2 N} \sum_{j=1}^{2 N}(-1)^{i+j} E\left(r_{i j}, z\right)$,

where $E\left(r_{i j}, z\right)$ is the magnetostatic interaction energy between two magnets in different sets. The magnet in the set of four has coordinates $(N, N)$, while the magnet in the underlying set has coordinates $(i, j)$. Parameters $r_{i j}$ and $z$ are their lateral and axial separation between the two magnet's centers, respectively. Their lateral displacement, $r_{i j}$ is

$r_{i j}=2 R \sqrt{(N-i)^{2}+(N-j)^{2}}$

According to Eq. (1), the axial attraction force between the two groups can be written as

$$
\begin{aligned}
F_{z}^{4 / 4 N^{2}}(\zeta, \tau)= & -4 F_{0} \int_{0}^{+\infty} \frac{d q}{q}\left\{\sum_{i=1}^{2 N} \sum_{j=1}^{2 N}(-1)^{i+j} J_{0}\left[2 q \sqrt{(N-i)^{2}+(N-j)^{2}}\right]\right\} \\
& \times J_{1}^{2}(q) \sinh ^{2}(q \tau) e^{-q \varsigma}
\end{aligned}
$$

If $N=1$, then Eq. (4) transforms into

$F_{z}^{4 / 4}=-4 F_{0} \int_{0}^{+\infty} \frac{d q}{q}\left[1-2 J_{0}(2 q)+J_{0}(2 \sqrt{2} q)\right] J_{1}^{2}(q) \sinh ^{2}(q \tau) e^{-q \varsigma}$

Eq. (5) is identical with Eq. (2) of [11] since the case $N=1$ reduces to the case studied in [11].

\subsection{The $N \rightarrow \infty$ limit}

Eq. (4) becomes impractical if $N$ is large. Therefore, to examine the scenario where one of the arrays contains a very large number of magnets, we chose a different approach. According to [12] (see also the Appendix), in the $N \rightarrow \infty$ limit the force becomes

$F_{z}^{4 / \infty}(\zeta, \tau)=-8 \pi F_{0} \sum_{i, j>0}^{o d d} \frac{J_{1}^{2}\left(\rho_{i j}\right)}{\rho_{i j}^{2}} \sinh ^{2}\left(\tau \rho_{i j}\right) e^{-\zeta \rho_{i j}}$

where

$\rho_{i j}=\frac{\pi}{2} \sqrt{i^{2}+j^{2}}$

As a further generalization of the model, we now allow the finite set of magnets to comprise $2 L \times 2 L$ cylindrical magnets, where $L=1,2,3, \ldots$ In this case, the force between a set of $2 L \times 2 L$ magnets and an infinite set, $F_{z}^{4 L^{2} / \infty}(\zeta, \tau)$, is simply $L^{2}$ times the force between four magnets and an infinite set

$F_{z}^{4 L^{2} / \infty}(\zeta, \tau)=L^{2} F_{z}^{4 / \infty}(\zeta, \tau)$

As mentioned in the introduction, the resulting attraction contact force between the two sets of $2 \times 2$ magnets can be larger than four times the attraction contact force between just two 


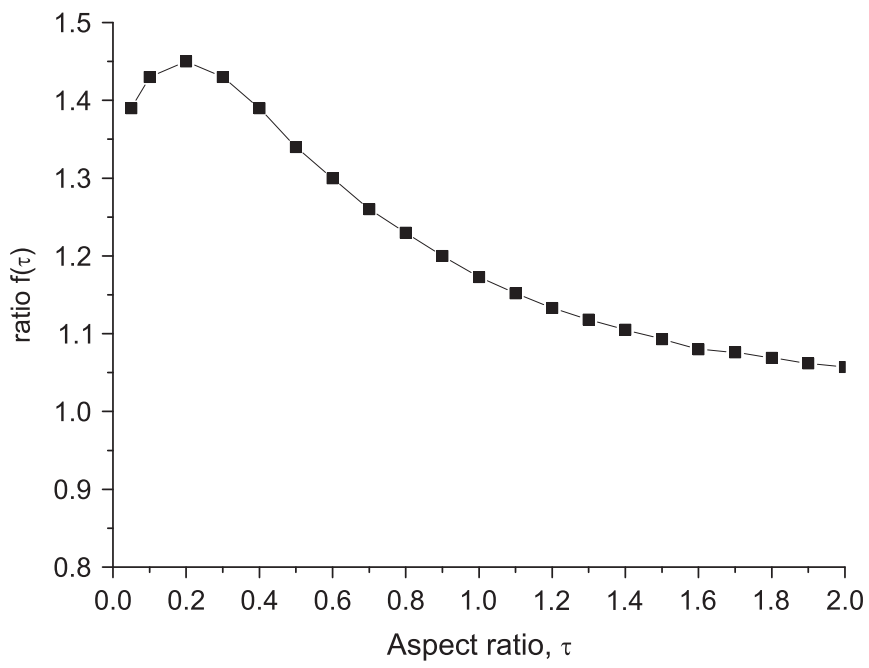

Fig. 2. The ratio $f(\tau)$ versus magnet aspect ratio $\tau$.

magnets. In general, let us assume two arrays, $2 L \times 2 L$ and $2 N \times 2 N$ where $N \rightarrow \infty$. We define a force ratio $f(\tau)$ as follows:

$f(\tau)=\left|\frac{F_{z}^{4 L^{2} / \infty}(2 \tau, \tau)}{4 L^{2} F_{z}^{1 / 1}(0,2 \tau, \tau)}\right|=\left|\frac{F_{z}^{4 / \infty}(2 \tau, \tau)}{4 F_{z}^{1 / 1}(0,2 \tau, \tau)}\right|$

As visible in Fig. 2, $f(\tau)$ has a non-monotonic behavior with a maximum (1.45) at $\tau=0.2$. As the aspect ratio increases, the force ratio converges to the value of 1 , as expected. This force ratio may be a useful parameter to keep in mind when tailoring magnetic arrays for fastener applications. However, when thinking of different applications, one should also consider that in the studied sets with periodically changing magnetization orientation, the attraction force decreases fast with increasing distance between two sets of magnets as shown in Ref. [11].

As already mentioned, the anti-parallel magnetization arrangement within the arrays is advantageous in that the magnets do not repel each other. However, if we overcome the repulsion force and form arrays $2 \mathrm{~N} \times 2 \mathrm{~N}$ and $2 \times 2$ using identical magnets aligned in parallel, and if $N \rightarrow \infty$ while we maintain all other assumptions we considered previously, then the attraction force between the two sets is calculated according to Eq. (6) but summing over even indexes $i, j$. We denote the attraction force between sets in parallel alignment by $F_{z}^{4 * / \infty *}(\zeta, \tau)$.

\section{Experimental setup}

To validate our theoretical framework, we carry out a set of experiments to measure the attraction force between various sets of magnets as a function of distance, and compare the results with predictions from the expressions we have developed. We use NdFeB permanent magnets purchased directly from their manufacturer Magsy s.r.o. Our measurement of magnetic induction with a gaussmeter (F.W. Bell, Model 6010) and Hall-effect probe (STD610202-05) gives a value, $B=(1.032 \pm 0.008) \mathrm{T}$, corresponding to a saturation magnetization $M=(0.821 \pm 0.006) \mathrm{MA} / \mathrm{m}$. The magnets are shaped as cylinders with a radius $R=2 \mathrm{~mm}$ and a height $t=8 \mathrm{~mm}$. They are magnetized axially by the producer. The magnets are coated with a thin layer of Ni to prevent oxidation. The samples are produced as follows: (i) either square array of magnets $(6 \times 6$ or $2 \times 2)$ is put onto a flat substrate surrounded by an $\mathrm{Al}$ frame; (ii) the magnets are glued together by hardened resin poured into the frame before a polymerization; (iii) after resin hardening, substrates are removed.
The attraction force measurements are carried out at room temperature with a tensile testing machine equipped with a $100 \mathrm{~N}$ load cell (HBM, Type S2). Great attention is paid to clamping magnet samples and their alignment since a small misalignment may result in discrepancy between measured and calculated force values. While one of the samples is firmly clamped the other sample is placed on the clamped magnet and glued to the free clamp. During the process of applying the glue, the measured force is zero. A non-negligible force develops during glue hardening (ideally, the force should remain zero). However, this force is smaller than that in the case when the other magnet sample is fixed by screws.

\section{Comparison of the experimental and calculated data}

Fig. 3 shows measured and calculated (according to Eq. (4) for $N=3$ ) data for the attraction force between two magnet sets $(2 \times 2)$ and $(6 \times 6)$ for various vertical displacements. Due to some small misalignment and the final rigidity of the clamps we are not able to measure the contact force accurately. The offset of Fig. 3 shows the large difference between the measured and the calculated contact force. We see that the measured force values are systematically smaller than those calculated. Excluding the data point for the contact force, the difference between the measured and calculated attraction forces is always below $10 \%$ (see Table 1), as their ratio ranges between 0.96 (gap $0.1 \mathrm{~mm}$ ) and 0.91 (gap $2 \mathrm{~mm}$ ). Since the ratio between measured and calculated values is not constant, we cannot explain the discrepancy with a simple rescaling of some quantity, either the magnets' field or its volume. The effect is, most likely, related to the actual shape of the magnets, which differs from an ideal cylinder in the rounded edges. The effective aspect ratio of the cylinders may also be a little different from the value we set $(\tau=2)$ since there may be a layer of non-magnetic material (or magnetic but with different material parameters) coating the lateral surfaces. Finally, non-uniformity effects are also very likely to play a role. In particular, it is reasonable to imagine that when the two sets are in proximity, interaction fields are effectively enhancing the alignment of the magnetic moments, resulting in a better uniformity, while when magnets are far apart, the degree of uniformity decreases.

To investigate further what effects the magnet coatings may have on our results, we peeled off the Ni layer covering our NdFeB magnets

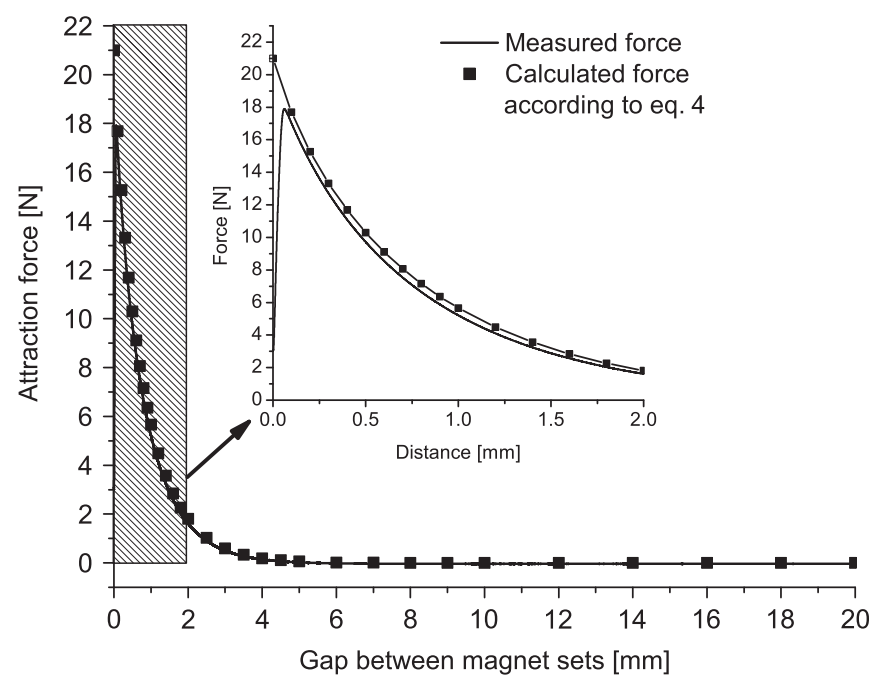

Fig. 3. Experimental and calculated (according to Eq. (4)) data for attraction force between two magnet sets $(2 \times 2)$ and $(6 \times 6)$. 
Table 1

A comparison of measured and calculated attraction force values according to various formulas and for various distances between the two sets of magnets.

\begin{tabular}{|c|c|c|c|c|}
\hline $\begin{array}{l}\text { Distance between the } \\
\text { magnet sets }(\mathrm{mm})\end{array}$ & $\begin{array}{l}\text { The measured } \\
\text { force }(\mathrm{N})\end{array}$ & $\begin{array}{l}\text { The calculated force } \\
\text { according to Eq. (4) (N) }\end{array}$ & $\begin{array}{l}\text { The calculated force } \\
\text { according to Eqs. (6) } \\
\text { and }(7)(N)\end{array}$ & $\begin{array}{l}\text { The calculated force according } \\
\text { to Eqs. (6) and ( } 7 \text { ) with } i=j=1 \\
\text { (only one addend) (N) }\end{array}$ \\
\hline 0 & $3 \pm 0.03$ & $21.00 \pm 0.32$ & $21.26 \pm 0.32$ & $16.57 \pm 0.25$ \\
\hline 0.1 & $17.05 \pm 0.17$ & $17.69 \pm 0.27$ & $17.71 \pm 0.27$ & $14.83 \pm 0.22$ \\
\hline 0.2 & $14.68 \pm 0.15$ & $15.27 \pm 0.23$ & $15.27 \pm 0.23$ & $13.27 \pm 0.20$ \\
\hline 0.3 & $12.74 \pm 0.13$ & $13.32 \pm 0.20$ & $13.32 \pm 0.20$ & $11.88 \pm 0.18$ \\
\hline 0.4 & $11.13 \pm 0.11$ & $11.69 \pm 0.18$ & $11.69 \pm 0.18$ & $10.63 \pm 0.16$ \\
\hline 0.5 & $9.76 \pm 0.10$ & $10.30 \pm 0.15$ & $10.30 \pm 0.15$ & $9.51 \pm 0.14$ \\
\hline 0.6 & $8.59 \pm 0.09$ & $9.11 \pm 0.14$ & $9.11 \pm 0.14$ & $8.51 \pm 0.13$ \\
\hline 0.7 & $7.57 \pm 0.08$ & $8.07 \pm 0.12$ & $8.07 \pm 0.12$ & $7.62 \pm 0.11$ \\
\hline 0.8 & $6.69 \pm 0.07$ & $7.16 \pm 0.11$ & $7.16 \pm 0.11$ & $6.82 \pm 0.10$ \\
\hline 0.9 & $5.92 \pm 0.06$ & $6.36 \pm 0.10$ & $6.36 \pm 0.10$ & $6.10 \pm 0.09$ \\
\hline 1.0 & $5.25 \pm 0.05$ & $5.66 \pm 0.08$ & $5.66 \pm 0.08$ & $5.46 \pm 0.08$ \\
\hline 1.2 & $4.15 \pm 0.04$ & $4.49 \pm 0.07$ & $4.49 \pm 0.07$ & $4.37 \pm 0.07$ \\
\hline 1.4 & $3.28 \pm 0.03$ & $3.57 \pm 0.05$ & $3.57 \pm 0.05$ & $3.50 \pm 0.05$ \\
\hline 1.6 & $2.61 \pm 0.03$ & $2.84 \pm 0.04$ & $2.84 \pm 0.04$ & $2.80 \pm 0.04$ \\
\hline 1.8 & $2.07 \pm 0.02$ & $2.27 \pm 0.03$ & $2.27 \pm 0.03$ & $2.24 \pm 0.03$ \\
\hline 2.0 & $1.65 \pm 0.02$ & $1.81 \pm 0.03$ & $1.81 \pm 0.03$ & $1.80 \pm 0.03$ \\
\hline
\end{tabular}

and measured its thickness. As the mean measured thickness was $27 \pm 14 \mu \mathrm{m}$, the average Ni content of our magnets was about $3 \%$ in volume. According to Ref [14], the saturation magnetization of $\mathrm{Ni}$ is about $0.6 \mathrm{~T}$, which is smaller than the saturation magnetization of $\mathrm{NdFeB}$ as measured and described in part 4. In order to ascertain whether or not the presence of a thin Ni layer might influence our measurement of the NdFeB magnetization, we repeated the measurements on magnets with and without $\mathrm{Ni}$ coating, finding only a negligible influence within statistical errors. Taking also into account the non-uniformity of the magnetization distribution in the Ni layer, we conclude that the presence of Ni coating most likely results in a slight decrease of the measured attraction force. Consequently, the calculations, carried out neglecting coatings, predict higher values for the attraction forces, which might explain the systematic theoretical overestimates visible in the comparative Table 1.

Another interesting point is that if we use Eq. (6) for the $4 \times 4$ case we obtain values close to those obtained from Eq. (4) for $6 \times 6$ and $2 \times 2$ (see Table 1 ). This means that in the case of our magnets $(\tau=2)$ the increasing size of the larger set does not matter too much for the value of the attraction force. This is most likely a consequence of the rapid decay of the field generated by the outer regions of the larger set, decay established by the antiparallel alignment of magnets. Furthermore, for $\tau=2$, the sum in Eq. (6) converges fast since the first addend approximates the attraction force relatively well (Table 1 ).

It is worth noting that if we compare the anti-parallel and parallel magnetization arrangement in the case $2 \times 2$ over $2 \mathrm{~N} \times 2 \mathrm{~N}$ where $N \rightarrow \infty$ and $\tau=2, M=0.821 \mathrm{MA} / \mathrm{m}$ then using Eq. (6) summing over $i, j$ both either odd (anti-parallel) or even (parallel), we obtain the following contact forces $\left|F_{z}^{4 / \infty}(4,2)\right|=21.26 \mathrm{~N}$ and $\left|F_{z}^{4 * / \infty *}(4,2)\right|=$ $1.83 \mathrm{~N}$. The contact force between two magnet arrays is defined as the attraction force with zero axial gap between the arrays $(\zeta=2 \tau)$. The contact force between anti-parallel magnets is much stronger than that of parallel magnets. This is a direct consequence of the slow decay of the magnetic field generated by the infinite array of parallel magnets, which acts on the north and south poles of the small set very differently than in the anti-parallel case. On the other hand, if we compare the $4 / 4$ cases $(2 \times 2$ over $2 \times 2)$, contact forces are comparable: $\left|F_{z}^{4 / 4}(4,2)\right|=20.92 \mathrm{~N}$ and $\left|F_{z}^{4 * / 4 *}(4,2)\right|=17.87 \mathrm{~N}$.

\section{Magnet sets versus single magnets}

In the context of magnetic fasteners, it may be interesting to assess whether arrays or single magnets are preferable. To this

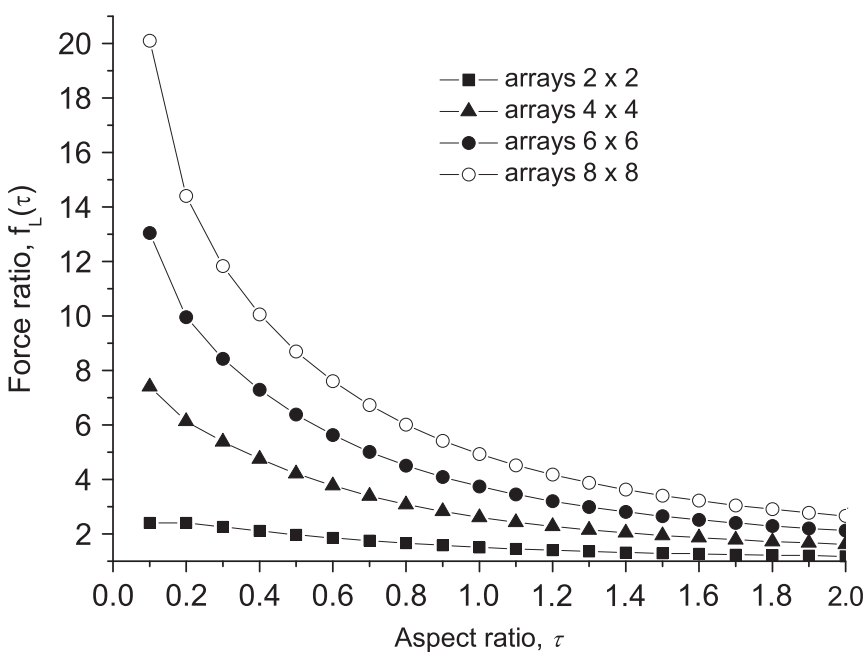

Fig. 4. Force ratio $f_{L}(\tau)$ or its estimate, versus aspect ratio $\tau$.

purpose, we analyze the contact force between two sets of $2 L \times 2 L$ magnets, and the contact force between two single cylindrical magnets, where we choose the thicknesses and radii of the single magnets in such a way to conserve the total volume of magnetic material as well as the contact surface area. This implies that $t^{\prime}=t$ and $R^{\prime}=2 L R$, and therefore a factor $4 L^{2}$ is needed to rescale $F_{0}$. We then define the ratio $f_{L}(\tau)=F_{1} / F_{2}$ where $F_{1}\left[F_{2}\right]$ is the contact force attributed to the magnet sets [single magnets].

$$
\begin{aligned}
f_{L}(\tau) & =\frac{F_{z}^{4 L^{2} / 4 L^{2}}(2 \tau, \tau)}{4 L^{2} F_{z}^{1 / 1}(0, \tau / L, \tau / 2 L)} \sim \frac{F_{z}^{4 / \infty}(2 \tau, \tau)}{4 F_{z}^{1 / 1}(0, \tau / L, \tau / 2 L)} \\
& =\frac{2 \pi \sum_{i, j>0}^{o d d} \frac{J_{1}^{2}\left(\rho_{i j}\right)}{\rho_{i j}^{2}} \sinh \left(\tau \rho_{i j}\right) e^{-2 \tau \rho_{i j}}}{\int_{0}^{\infty} \frac{J_{1}^{2}(q)}{q} \sinh ^{2}\left(\frac{q \tau}{2 L}\right) e^{-\frac{q \tau}{L}} d q}
\end{aligned}
$$

In the $L=1$ case we can use Eq. (5) for $F_{1}$. Fig. 4 shows the curves $f_{1}(\tau)$ as a function of the aspect ratio for $L=1,2,3,4$. It is clear that for large arrays and small aspect ratios, the contact force between two array sets of magnets is much larger than the contact force between two cylindrical magnets of equivalent volume and contact area. 


\section{Conclusion}

We have reviewed formulas for calculating attraction forces between two cylindrical permanent magnets or two sets of permanent magnets and additionally, we derived the formulas for more general case of cylindrical permanent magnets of various radii and heights. For all the formulas we assumed (i) uniform magnetization in each cylinder and (ii) parallel magnetization directions and magnet axes.

The attraction force measurements were performed at room temperature using two sets of magnets consisting of $2 \times 2$ and $6 \times 6 \mathrm{NdFeB}$ magnets with a diameter of $4 \mathrm{~mm}$ and a height of $8 \mathrm{~mm}$. In both samples the magnets were arranged in square arrays in such a way that magnetization vectors of any two closest magnet neighbors were oriented anti-parallel. The calculated force overestimated the measurements by less than $10 \%$. The rather large discrepancy between the measured and the calculated contact forces (Table 1 ) may be explained by a combination of: (i) misalignment of sets when measuring forces; (ii) spread in the saturation magnetization of each individual magnet, including the influence of $\mathrm{Ni}$ coating; (iii) the assumption of uniform magnetization in the cylindrical magnets. For large arrays and small aspect ratios, the contact force between two array sets of magnets is much larger than the contact force between two cylindrical magnets of equivalent volume and contact area.

\section{Acknowledgements}

DV acknowledges support by a Marie Curie International Reintegration Grant within the 6th European Community Framework Programme and by the Grant Agency of the Czech Republic under contract no. 101/09/0702. GT acknowledges support from the Italian INDAM-GNFM through project: "Modellazione fisico-matematica di materiali e strutture intelligenti (Smart Material Modeling)".

\section{Appendix}

In this appendix we illustrate how to calculate the interaction energy between two magnet arrays: $2 \times 2$ and $2 N \times 2 N$, where $N \rightarrow \infty$. We refer to [12] for additional details. The force can be obtained by differentiating the energy with respect to the axial coordinate. Calculations are carried out in Fourier space according to the formalism described, e.g., in Ref. [15].

Within a Cartesian reference frame $\left\{\mathbf{e}_{x}, \mathbf{e}_{y}, \mathbf{e}_{z}\right\}$, the magnetization of a cylindrical magnet of radius $R$ and height $2 d$ with its center in the origin can be written as

$\mathbf{M}_{1}(\mathbf{r})=M D_{1}(\mathbf{r}) \mathbf{e}_{z}$,

where $D_{1}(\mathbf{r})$ is the shape function, whose value is 1 inside the cylinder and 0 outside it. The function $\mathbf{M}_{4}(\mathbf{r})$ defined as

$\mathbf{M}_{4}(\mathbf{r})=\sum_{i, j \in\{-1,1\}}(-1)^{i+j} \mathbf{M}_{1}\left(\mathbf{r}-\operatorname{Ri}_{x}-R j \mathbf{e}_{y}\right)$

expresses the magnetization of four antiparallel axially magnetized cylinders with their centers in $( \pm R, \pm R, 0)$. It is convenient to introduce a function $D_{4}(\mathbf{r})$ :

$D_{4}(\mathbf{r})=\sum_{i, j\{-1,1\}}(-1)^{i+j} D_{1}\left(\mathbf{r}-R i \mathbf{e}_{x}-R j \mathbf{e}_{y}\right)$

that, strictly speaking, is not a shape function. However the following relationship holds:

$\mathbf{M}_{4}(\mathbf{r})=M D_{4}(\mathbf{r}) \mathbf{e}_{z}$

so that $D_{4}(\mathbf{r})$ can be interpreted as an effective shape function. In the set of $2 \mathrm{~N} \times 2 \mathrm{~N}$ magnets, the unit of the four magnets with magnetization characterized by $\mathbf{M}_{4}(\mathbf{r})$ is periodically repeated, with periodicity $4 R$. Therefore we can express the effective shape function of the whole $2 N \times 2 N$ array where $N \rightarrow \infty$ as

$D_{\infty}(\mathbf{r})=\sum_{i, j=-\infty}^{\infty} D_{4}\left[\mathbf{r}-4 R\left(i \mathbf{e}_{x}-j \mathbf{e}_{y}\right)\right]$

Finally, the second set of $2 \times 2$ magnets, is characterized by the effective shape function $D_{2} \times{ }_{2}(\mathbf{r})=D_{4}\left(\mathbf{r}-Z \mathbf{e}_{z}\right)$, where $Z$ is the vertical distance between the two arrays' centers.

We denote Fourier-transformed quantities by a superimposed hat and indicate a position vector in Fourier space with $\mathbf{k}=\left(k_{x}, k_{y}, k_{z}\right)$. We further define $k_{\perp}=\sqrt{k_{x}^{2}+k_{y}^{2}}$. The shape amplitude of a single cylinder is [16]

$\hat{D}_{1}(\mathbf{k})=2 V \frac{J_{1}\left(R k_{\perp}\right)}{R k_{\perp}} \frac{\sin \left(d k_{z}\right)}{d k_{z}}$

where $V=2 \pi R^{2} d$ is the volume of the cylinder. According to the general properties of Fourier transforms, we have

$\hat{D}_{4}(\mathbf{k})=-4 \sin \left(R k_{x}\right) \sin \left(R k_{y}\right) \hat{D}_{1}(\mathbf{k})$

$\hat{D}_{2 \times 2}(\mathbf{k})=\exp \left(i Z k_{z}\right) \hat{D}_{4}(\mathbf{k})$

$\hat{D}_{\infty}(\mathbf{k})=\left(\frac{\pi}{2 R}\right)^{2} \sum_{i, j=-\infty}^{+\infty} \hat{D}_{4}\left(\frac{i \pi}{2 R}, \frac{j \pi}{2 R}, k_{z}\right) \delta\left(k_{x}-\frac{i \pi}{2 R}\right) \delta\left(k_{y}-\frac{j \pi}{2 R}\right)$

The magnetostatic interaction energy between two bodies with magnetizations $M_{\mathrm{A}}$ and $M_{\mathrm{B}}$ can be conveniently expressed as

$E_{i}=\mu_{0} \operatorname{Re}\left(\mathfrak{J}^{-1}\left\{\frac{\left[\hat{M}_{A}(\mathbf{k}) \cdot \mathbf{k}\right]\left[\hat{M}_{B}^{*}(\mathbf{k}) \cdot \mathbf{k}\right]}{k^{2}}\right\}\right)$

where $\mathfrak{J}^{-1}$ is the inverse Fourier transform operator, and the asterisk * denotes complex conjugation. For our geometry, the energy integral is

$E_{i}=\operatorname{Re}\left(\frac{\mu_{0} M^{2}}{8 \pi^{3}} \int \frac{k_{z}^{2}}{k_{z}^{2}+k_{\perp}^{2}} \hat{D}_{\infty}(\mathbf{k}) \hat{D}_{2 \times 2}(\mathbf{k}) \mathrm{d}^{3} \mathbf{k}\right)$

which, after introducing $\rho_{i j} \equiv \frac{\pi}{2} \sqrt{i^{2}+j^{2}}$ and $\tau=d / R$, evaluates to

$E_{i}=\frac{2 \mu_{0} M^{2}}{\pi}\left(\frac{V}{R}\right)^{2} \sum_{i, j=-\infty}^{+\infty} \frac{J_{1}^{2}\left(\rho_{i j}\right)}{\rho_{i j}^{2}} \sin ^{2}\left(i \frac{\pi}{2}\right) \sin ^{2}\left(j \frac{\pi}{2}\right) \int_{-\infty}^{\infty} \frac{\sin ^{2}\left(d k_{z}\right) \cos \left(Z k_{z}\right)}{\left(d k_{z}\right)^{2}+\tau^{2} \rho_{i j}^{2}} d k_{z}$

The integral along $k_{z}$ can be carried out explicitly, yielding

$E_{i}=32 \mu_{0} \pi^{2} M^{2} R^{3} \sum_{i, j=1}^{\infty} \frac{J_{1}^{2}\left(\rho_{i j}\right)}{\rho_{i j}^{3}} \sin ^{2}\left(i \frac{\pi}{2}\right) \sin ^{2}\left(j \frac{\pi}{2}\right) \sinh ^{2}\left(\tau \rho_{i j}\right) e^{-\zeta \rho_{i j}}$

from which the force can be derived as in Eq. (6) by direct differentiation with respect to $Z=\zeta R$.

In case of parallel alignment of all magnets involved (both sets), the energy is as in (A11) with the exception of the two $\sin ^{2}$ terms that are replaced by $\cos ^{2}$. As a consequence, the sum is evaluated with even-only indexes (as opposed to odd-only indexes when magnets are anti-parallel).

\section{References}

[1] B. Ebrahimi, M.B. Khamesee, F. Golnaraghi., Microsyst. Technol. 16 (2010) 19-24.

[2] J.B. Nestleroth, R.J. Davis., NDT \& E International 40 (2007) 77-84.

[3] S.J. Lee, J.M. Kenkel, V.K. Pecharsky, D.C. Jiles., J. Appl. Phys. 91 (2002) 8894-8896.

[4] X.N. Xu, D.W. Lu, G.Q. Yuan, Y.S. Han, X. Jin., J. Appl. Phys. 95 (2004) 6302-6307.

[5] Y. Su, W. Chen, W. Zhang., Int. J. Adv. Manuf. Technol. 30 (2006) 215-220.

[6] J. Lugo, V. Sosa., Physica C 324 (1999) 9-14. 
[7] U.O. Hafeli, K. Gilmour, A. Zhou, S. Lee, M.E. Hayden., J. Magn. Magn. Mater. 311 (2007) 323-329.

[8] M. Golosovsky, Y. Saado, D. Davidov, Appl. Phys. Lett. 75 (1999) 4168-4170.

[9] S.B. Shetye, J.A. Agashe, D.P. Arnold., IEEE trans. Magn. 43 (2006) 2713.

[10] O.J. Suarez, P. Vargas, E.E. Vogel, J. Magn. Magn. Mater. 321 (2009) 3658.

[11] D. Vokoun, M. Beleggia, L. Heller, P. Sittner., J. Magn. Magn. Mater. 321 (2009) 3758-3763.

[12] G. Tomassetti, Open archive of Università degli Studi di Roma "Tor Vergata", URI:〈http://dspace.uniroma2.it/dspace/handle/2108/1186〉.
[13] M. Abramowitz, I. Stegun, Handbook of Mathematical Functions, Dover, New York, 1970 , p. 360.

[14] B.D. Cullity, Introduction to Magnetic Materials, Addison-Wesley Publishing Company, 1972.

[15] M. Beleggia, S. Tandon, Y. Zhu, M. De Graef., J. Magn. Magn. Mater. 278 (2004) 270-284

[16] S. Tandon, M. Beleggia, Y. Zhu, M. De Graef., J. Magn. Magn. Mater. 271 (2004) $9-26$. 